Review

\title{
Route to OCT From OFS at University of Kent
}

\author{
Adrian PODOLEANU \\ Applied Optics Group, School of Physical Sciences, University of Kent, Canterbury, CT2 7NH, UK \\ *Corresponding author: Adrian PODOLEANUＥ-mail: ap11@kent.ac.uk
}

\begin{abstract}
A review is presented of several technical solutions developed by the Applied Optics Group (AOG) in the field of low coherence interferometry applied to optical fiber sensors (OFS) that subsequently allowed AOG to quickly progress in the field of optical coherence tomography (OCT).
\end{abstract}

Keywords: Low coherence interferometry, optical coherence tomography, eye imaging

\section{Introduction}

Several concepts of low coherence interferometry (LCI) derived from fiber optic sensing, fiber lasers, and fiber optic communications, developed by the Applied Optics Group (AOG) will be presented along with their adaptation to imaging methods of tissue where the main driving force was resolution improvement. References will be made to selected published work by several members of the AOG over the years, which represent key advancements towards optical coherence tomography (OCT). By the time they were developed, OCT was either inexistent or in its infancy. In other words, the motivation in progressing the research was totally different from what we mean by the OCT field today. However, the achievements reviewed here represent milestones in building up the expertise that conditioned the later rapid developments within the AOG towards principles, optical sources and signal processing specific to OCT. References will then be made to the results that the AOG has subsequently obtained in the OCT field. To keep this review within limits, a limited number of references from outside AOG are mentioned only. The interested reader in a particular subject, could however follow the complete list of references in each report published by AOG and mentioned here.

Modern OCT technology uses a specialized optical source, such as a broad band source or a tunable source within a broad band, in both cases exceeding several tens of nanometers. OCT also makes use of an interferometer and a scheme for signal processing. All these elements have been in some form approached by the AOG during research on single mode fiber optic sensors [1]. Such research, as early as 1982, used a Mach-Zehnder interferometer, a phase modulator, and two detectors in a balanced configuration. Later, a novel form of phase modulation will be proposed by AOG, employing the optical path variation that takes place when a transversal scanner [2] is employed to scan a target laterally. Bessel function decomposition, widely used in signal processing of white light interferometer configurations in the optical fiber sensors (OFS) field will also be utilized to enhance the OCT signal produced by the path modulation. Two band pass filters, tuned on two harmonics within the broad bandwidth spectrum created by path modulation lead to enhancement of signal to noise $(\mathrm{S} / \mathrm{N})$ ratio [3].

Received: 26 October 2010 / Revised version: 28 November 2010

(C) The Author(s) 2011.This article is published with open access at Springerlink.com 
This is one example whereby conducting research into sensing in general and in OFS in particular, the AOG contributed towards several directions which subsequently paved the way towards the later development of what is known today as OCT. Other examples include the evaluation of heterodyne [4, 5] principles, at the core of demodulation schemes used in time domain OCT and in elimination of mirror terms in spectral OCT. Early research within the AOG was performed using methods which now can be attributed to the core of all three types of OCT: 1) time domain (essentially a Michelson interferometer), 2) spectral or Fourier domain (FD), where the interferometer output is sent to an optical spectrometer, and 3) swept source (SS), where the frequency of a narrowband optical source is swept within a broad band.

Essential work in the early phase of low coherence interferometry was to understand its advantage in terms of absolute path and phase measurement. Such studies have shown that a sensor employing low coherence interferometry has the full resolution of a conventional interferometer, but has the major advantage of an unambiguous operating point [6].

As another example, elements useful for what is now known as common path [7] OCT, have been equally approached, such as using the cladding mode to act as reference in a common path interferometer [8]. More examples are from the field of tandem interferometers [9] and signal processing for such sensing configurations [10], common path heterodyne LCI [11], polarization insensitive tandem [12] and Fizeau interferometer with a broadband source, such as a super-fluorescent source [13].

Manipulation of polarization $[14,15]$ was established in detail empowering AOG with essential skills for future assembling of complex single mode fiber OCT configurations.

Piezo modulation [16], needed for phase shifting interferometry in full field OCT was intensely investigated during research on OFS.

Dispersion is a major problem for low coherence interferometry (and OCT), if left uncompensated, the capability of resolving quantities measured along optical path suffers in the same way as the axial resolution suffers in OCT. Measurement of dispersion in a short fiber length [17] was reported and this represents a precursor of similar measurement performed today with ultra large bandwidth optical sources.

Multimode laser diodes [18, 19] were used as early low coherence sources, precursors of super-luminescent diodes that are nowadays largely used in the modern OCT technology.

Knowledge on how the properties of optical sources affect the measurement resolution has been acquired in the process of research on coherence synthesized optical sources [20].

The concept of a broadband source followed by spectral filtering [21] was the first type swept source OCT, concept also approached by AOG [22] as a fiber sensing mean.

\section{AOG contributions to TD-OCT}

There are two main LCI and OCT methods, time domain (TD) and spectral domain (SD).

A reflectivity profile in depth (A-scan) is produced by scanning the optical path difference (OPD) in the interferometer. A transversal reflectivity profile (T-scan) can also be produced by transversally scanning the beam over the target, i.e. by en-face ( $e F$ ) flying the spot while maintaining the reference mirror fixed. The lateral size could be scaled versus beam tilting angle or versus lateral displacement of the scanning beam. In both cases, of A-scanning and T-scanning, the interferometric signal is processed in time. The A-scan can be considered as a high axial resolution optical time domain reflectometry (OTDR) scan produced by an LCI setup. A cross section (B-scan) image can be obtained by grouping together several A-scans collected for different transversal positions or several $\mathrm{T}$-scans for different depth positions in the sample. 


\subsection{Sampling function in en-face OCT}

In order to generate constant depth (or en-face or C-scan) images at a fixed depth, a path imbalance modulator is needed so as to create a carrier for the image bandwidth. This will obviously require the introduction of a phase modulator in one of the arms of the interferometer, which would complicate the design of the interferometer and introduce dispersion. Research in AOG has shown that the $X$ or $Y$-scanning device itself introduces a path modulation which plays a similar role to the path modulation created by the longitudinal scanner employed to produce A-scans or A-scan based B-scans in conventional OCT, as presented in the first paper which introduced the OCT term [23]. Theoretical analysis has shown that the generation of a C-scan OCT image can be interpreted as interrogating the object with a specific sampling function. Depending on the position of the incident beam on the scanner mirror and on the interface optics used, the sampling function could look either as Newton rings [2] or as a regular grid of lines [24]. The sampling function is in fact a fringe pattern in transversal section. Consequently, when the beam scans the target, the OCT signal is modulated by this fringe pattern.

\subsubsection{Newton rings}

Figure 1 shows the beam being deflected from point $\mathrm{O}$ on the galvanometer mirror $\mathrm{MX}$ by tilting this mirror at different angles $\beta$ (i.e. while performing $e F$ scanning). The conjugate point of $\mathrm{O}$ is $\mathrm{O}^{\prime}$ and therefore the optical path lengths of all the reflected rays measured between mirror MX and $\mathrm{O}^{\prime}$ are equal. Let us consider a flat surface $\mathrm{S}$ as the object under test, perpendicularly oriented to the system axis and intersected by the scanned beam at $\mathrm{N}$.

The coherence surface $\Sigma$, defined by the length of the reference arm, is given by the spherical surface of radius $r$ centered in O'. For each scanning angle $\beta$, two beams are superposed on the photodetector, one being reflected from the point $\mathrm{P}$ on surface $\Sigma$ and the second being reflected by mirror S. Evaluating the OPD between the reference arm and the sensing arm leads to the geometrical locus of the points on the surface $\mathrm{S}$ of maximum interference. This locus is described by rings of radius $\mathrm{O} " \mathrm{~N}$. For a central wavelength $\lambda$, small angles and low number $M$ of rings, the radius of such rings is given by

$$
R_{M} \approx \sqrt{M r \lambda}
$$

which shows that the locus of maximum interference is given by a similar relation to that describing Newton rings [2]. However, the configuration in Fig. 1 differs from the Newton rings configuration presented in classical optics textbooks based on a spherical element in contact with a planar element. Here, the interfering rays producing Newton rings are coming from two different arms of the interferometer.

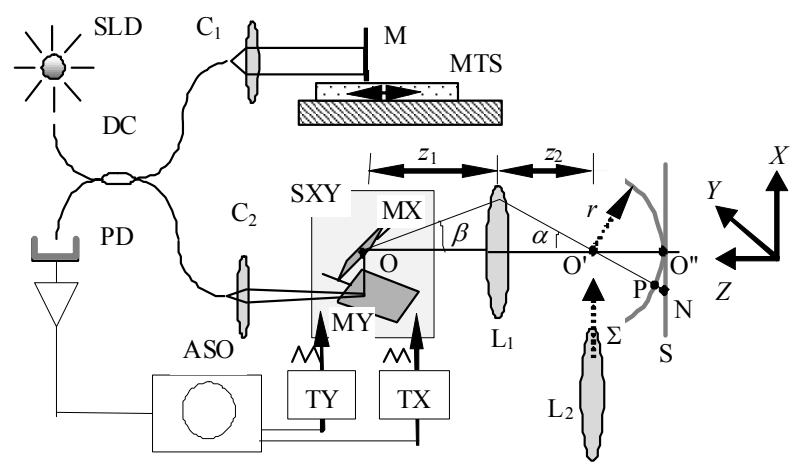

Fig. 1 Time domain en-face OCT configuration (SLD: superluminescent diode; $\mathrm{DC}$ : directional coupler; $\mathrm{C}_{1}, \mathrm{C}_{2}$ : microscope objectives; M: mirror; MTS: micrometer translation stage; SXY: galvanometer scanning mirror system; MX, MY: scanner mirrors; $\mathrm{L}_{1}, \mathrm{~L}_{2}$ : lenses; $\mathrm{PD}$ : photodetector; ASO: analogue storage oscilloscope; TX, TY: triangle waveform generators).

The analysis shows that the object is being interrogated with a sampling function which in the particular case of the setup in Fig. 1 looks like concentric circles centered on the system axis. An image from a mirror displays the sampling function pattern described by Newton rings, as shown in Fig. 2. Such an image was obtained by using a lens $\mathrm{L}_{2}$ of $2.5-\mathrm{cm}$ focal length, and by driving the horizontal and vertical scanners with signals at $F_{x}=20 \mathrm{~Hz}$ (signal generator GX) and $0.25 \mathrm{~Hz}$ (signal generator 
GY) respectively. The amplitude of both signals was $0.25 \mathrm{~V}$ and galvo-scanners with a conversion constant of $k=69.81 \mathrm{rad} / \mathrm{V}$ were used.

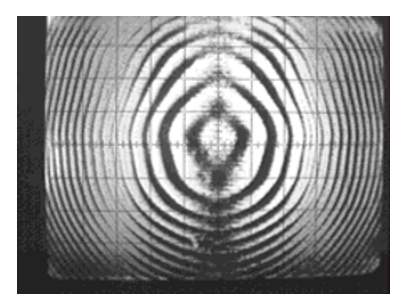

Fig. 2 Newton rings sampling function $(50 \mathrm{mV} / \mathrm{div}$ $(185 \mu \mathrm{m} / \mathrm{div}))$.

The main advantages of the method are its simplicity and quick display capability.

\subsubsection{Grid sampling function}

The incident beam direction is shifted by $\delta$ away from the rotation center of the line scanner, chosen to be the $X$-scanner in Fig. 3. For $\beta=0$, the incoming ray is incident on the galvanometer mirror in a point $\mathrm{B}$ on the optical axis (of the lens), situated at $z_{1}-\delta$ away from lens $\mathrm{L}_{1}$. Shown in Fig. 3 is a ray undergoing reflection at mirror MX when MX is at the angular position $\beta=0$ (impact point $\mathrm{B}$ ) and $\beta \neq 0$ (impact point $\mathrm{C}$ ). The ray deflected from $\mathrm{C}$ intersects the optical axis in $\mathrm{A}$. For small scanning angles $\beta$, the segment $\mathrm{AB} \approx \delta$. In these circumstances it can be shown that the path imbalance introduced between the central ray (deflected from $\mathrm{B}$ along the optic axis) and the ray deflected from $\mathrm{C}$ at an angle $\beta$ from the optic axis is given by addition of two terms, one linear in $\delta$ (arising from the double pass of light along segment $\mathrm{BC}$ to and from the object, and the other one proportional to $\delta^{2}$ (responsible for Newton rings imaging). For an amplitude voltage $U$ applied to the line scanner, $X$, a galvo-scanner conversion constant $k$ (degree/voltage), focal length of lens $L_{2}, f_{2}$ and distances $z_{1}$ and $z_{2}$ as shown in Figs. 1 and 3, it can be shown that during each scan, the frequency $v$ varies between

$$
v_{\text {min,max }}=v_{\mathrm{c}} \pm v_{\mathrm{n}}=\frac{8 k F_{x} U}{\lambda}\left[\delta \pm f_{2} \frac{z_{1}^{2}}{z_{2}^{2}} \beta_{m}\right]
$$

where $v_{\mathrm{c}}$ is the central frequency and $v_{\mathrm{n}}$ the frequency spread owing to the non-linear $O P D$ dependence on $\beta$. Interference occurs for $|O P D|<l_{\mathrm{c}}$, where $l_{\mathrm{c}}$ is the coherence length of the source, and the maximum angle $|\beta|$ for which $|O P D|=l_{\mathrm{c}}$ is $\beta_{\mathrm{c}}$. For $|\beta|$ $<\beta_{\mathrm{c}}, \beta_{\mathrm{m}}=\beta_{\mathrm{c}}$, and for $|\beta|>\beta_{\mathrm{c}}, \beta_{\mathrm{m}}=k U$. The linear term in (2) becomes dominant when the shift $\delta$ is high enough.

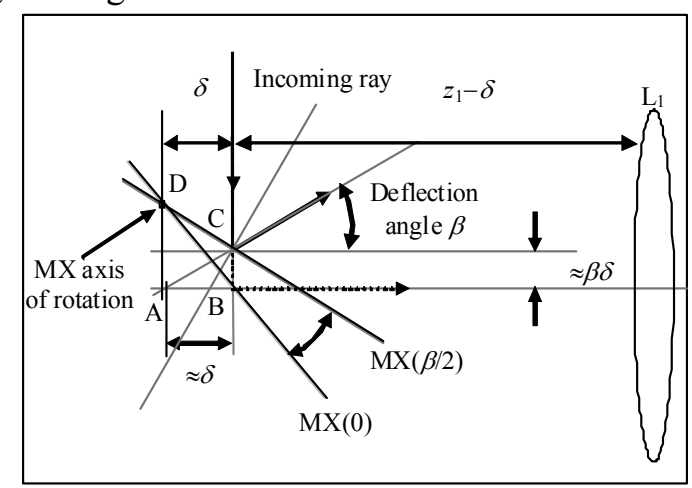

Fig. 3 Illustration of the incoming ray shifted away from the pivot of the line scanner in Fig. 1 (the object beam in Fig. 1 is incident on the galvanometer scanner at a distance $\delta$ away from the center of rotation; axes $X$ and $Z$ are in the plane of the drawing).

A "carrier" is distinguishable as shown in Fig. 4 left bottom (beam displaced by $\delta=3 \mathrm{~mm}$ ), in comparison with the non-displaced beam spectrum shown in Fig. 4 left top. For example, values of $v_{c}=$ $6.86 \mathrm{kHz}$ and $v_{\mathrm{n}}=1.6 \mathrm{kHz}$ are obtained from (2), in agreement with Fig. 4 bottom left, when $F_{x}=20 \mathrm{~Hz}$, $\lambda=0.83 \mu \mathrm{m}, D=2 \mathrm{~mm}, f_{2}=3 \mathrm{~cm}$, and $\Delta X=2 \mathrm{~mm}$.
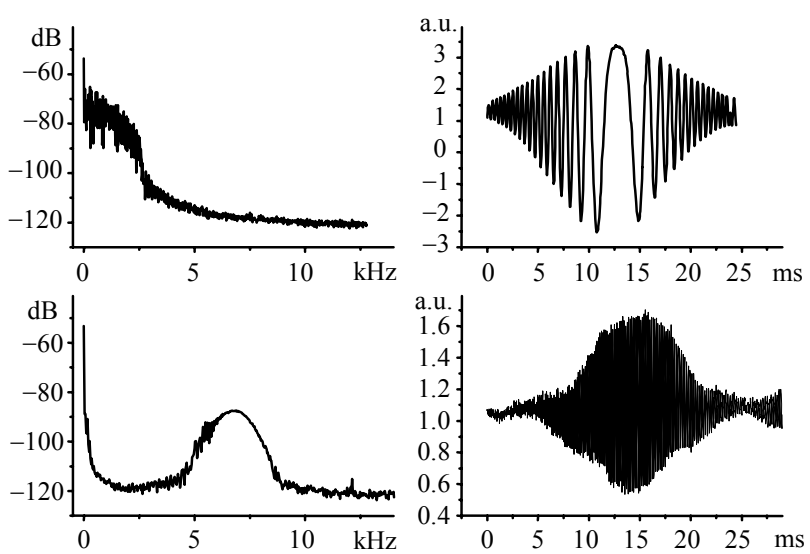

Fig. 4 Spectra (left) and the temporal evolution (right) for optical beam centered (top) and shifted by $\delta=3 \mathrm{~mm}$ (bottom) ( $z_{1}$ $=14.5 \mathrm{~cm}, z_{2}=10.2 \mathrm{~cm}$, a triangular driving signal of amplitude $U=0.17 \mathrm{~V}$ and frequency $F_{x}=20 \mathrm{~Hz}$ is applied to the $X$-scanner, while the $Y$-scanner is not driven). 
To produce a lateral scan $\Delta X=2 \mathrm{~mm}$ for $z_{1}=$ $14.5 \mathrm{~cm}$ and $z_{2}=10.2 \mathrm{~cm}$, the value of the applied voltage is $\mathrm{U}=0.34 \mathrm{~V}$. For $l_{\mathrm{c}} \sim 32 \mathrm{~m}$, in order for the first term to dominate in (2), it can be shown that $\delta$ should be higher than $2.1 \mathrm{~mm}$. The sampling function takes the form of a grid of regular lines, as shown in Fig. 5. This was obtained by driving the $X$ and $Y$ scanners with ramp signals of amplitude $0.17 \mathrm{~V}, F_{x}=20 \mathrm{~Hz}$ and $F_{y}=0.2 \mathrm{~Hz}$, for $\delta=3 \mathrm{~mm}$ and using the same scanner head as that used to generate the sampling function in Fig. 2.

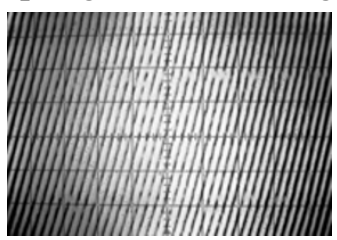

Fig. 5 Grid sampling function (horizontal and vertical scale: $10 \mathrm{mV} /$ div).

By driving the $X$ galvo-scanner with a ramp signal at a frequency $300 \mathrm{~Hz}$, amplitude $2 \mathrm{~V}$ and $\delta=$ $3 \mathrm{~mm}$ (maximum permissible by the size of the scanning mirror), a value of $v_{\mathrm{c}}$ in excess of $200 \mathrm{kHz}$ was obtained.

The sampling function acts as a selective topographic function, where the only object features that are displayed are those within the range $|\mathrm{OPD}|<l_{\mathrm{c}}$.

\subsection{OCT/SLO and OCT/SLO/ICG system}

The method of $e F-\mathrm{OCT}$ offers certain advantages. First, T-scans can be used to generate B-scans as well as coronal plane oriented scans (C-scans) [3]. This allows quick alternating imaging sessions in orthogonal planes by instantly switching scanning regimes $[25,26]$. Second, the technology is compatible with live and simultaneous generation of a conventional fundus image (coronal-plane oriented) $[27,28]$, or similar to that produced by scanning laser ophthalmoscopy (SLO). This also opens the avenue towards fluorescence imaging and the possibility of assembling multiple imaging channels in the same instrument.

Recently, the frequency shift introduced by placing the incidence point away form the pivot was suggested as a frequency shifting modality in a solution to eliminate the mirror terms in SD-OCT [29].

\subsubsection{OCT/SLO instrument}

Simultaneous acquisition of images in two channels, OCT and SLO, requires configurations using splitters. A small fraction of light returned from the retina is diverted towards the SLO channel using a separate splitter A dedicated AOG study investigated the optimum splitting ratio to ensure sufficient and similar $\mathrm{S} / \mathrm{N}$ ratio in both channels [30].

The main blocks of the $e F-\mathrm{OCT} / \mathrm{SLO}$ system are shown in Fig. 6. The scanning procedure is similar to that used in any SLO system, where the fast scanning is en-face (line rate, using the scanning mirror MX) and the frame scanning is much slower (at the frame rate, using the scanning mirror MY). The MX mirror is driven with a ramp at $500 \mathrm{~Hz}$ while the MY mirror is driven with a ramp at $2 \mathrm{~Hz}$. In this way, an en-face image in the plane $(x, y)$ is generated at constant depth. The next en-face image at a new depth is then generated by altering the length of the reference path of the OCT interferometer, by controlling a translation stage in the reference path and repeating the $(x, y)$ scan.

To construct B-scan images, the line scanner is driven with the same signal as in the $\mathrm{C}$-scanning regime and the translation stage modifies the length of the interferometer arm continuously over the designated depth range in $0.5 \mathrm{~s}$. In this case, an OCT cross-section image is produced either in the plane $(x, z)$ or $(y, z)$.

A case of macular pucker investigated using the OCT/SLO system is shown in Fig. 7.

The SLO image (Fig. 7(a) left) displays fine swirling striations on the macular surface. The corresponding $\mathrm{C}$-scan OCT image (Fig. 7(a) right) shows the tilted orientation of the retina, revealing the edge of the macular surface in oblique cross-section which highlights the infolding of the surface. The B-scan (Fig. 7(b)) shows the corrugated configuration of the retina due to the epiretinal membrane (ERM) and its effect on the middle layers as well as the surface. 


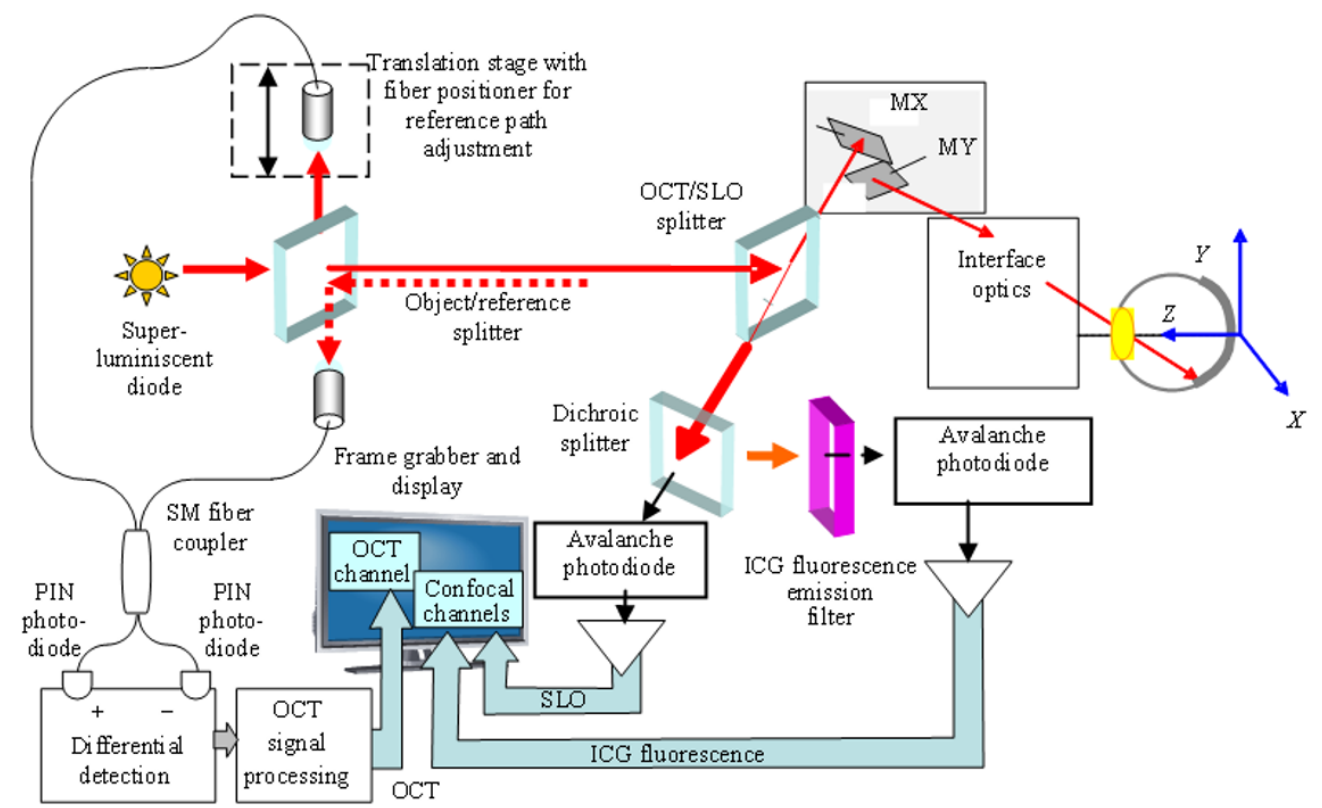

Fig. 6 Combined OCT/SLO/ICG (indocyanine green) system (MX, MY: galvanometer mirrors of the XY scanning pair, the confocal channels display a standard SLO image and an ICG fluorescence image (according to the paragraph 3 below)).

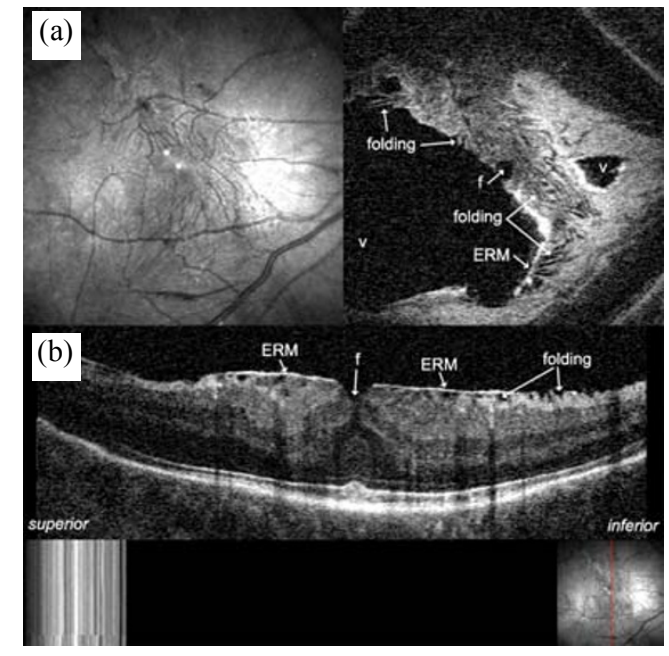

Fig. 7 Macular pucker [31]: (a) SLO image (left) and C-scan OCT image (right) and (b) B-scan OCT image (underneath the $\mathrm{B}$-scan image, there are $\mathrm{C}$-scan images, left, simultaneously acquired with the B-scan, while in the right, the $\mathrm{C}$-scan image obtained just before switching the system from the C-scan regime to the B-scan regime).

\subsubsection{Combination of OCT/SLO with fluorescence imaging}

On one hand, ICG angiography and OCT appear well suited to operate together because they share similar spectral bands. The most widely used band for retinal OCT is $820 \mathrm{~nm}$ to $920 \mathrm{~nm}$ while ICG is usually excited at $806 \mathrm{~nm}$ and fluoresces in the band $810 \mathrm{~nm}$ to $860 \mathrm{~nm}$ with a peak at $830 \mathrm{~nm}$. Operating in similar bands allows the same source to be used for ICG excitation as well as for the production of an OCT image.

A chromatic splitter [32] is used in Fig. 6 to divert some of the light to a separate confocal receiver. This separates the retina-scattered light at the excitation wavelength $792 \mathrm{~nm}$ guided into the OCT channel, from the fluorescence signal, centered at $830 \mathrm{~nm}$, guided towards the fluorescence confocal receiver. The residual transmission of the chromatic splitter (approx. 4\%) at the OCT wavelength is sufficient to generate an SLO image. The fluorescence signal and the residual signal at the excitation wavelength are separated by a second chromatic splitter. To enhance the contrast of fluorescence in the confocal receiver, a supplementary filter "fluorescence emission filter" is used in the fluorescence channel to attenuate any excitation band light that gets past the two chromatic splitters. These are cold mirrors with a transition wavelength $\lambda_{\text {rr }}$ between the excitation band and fluorescence band. Superlum Moscow developed a comparatively powerful SLD for this project, with 
an output power of $5 \mathrm{~mW}$ ex fiber at $\lambda=792 \mathrm{~nm}$ and $\Delta \lambda=21 \mathrm{~nm}$ spectral full width half maximum (FWHM) which determines a depth resolution in the tissue in the OCT channel of less than $9 \mu \mathrm{m}$ (considering an index of refraction $n \approx 1.4$ ).

The eF-OCT/fluorescence concept was also extended to microscopy, where the fluorescence channel was tuned on the fluorescence of green fluorescein protein and excitation was produced with a separate laser [33].

\subsection{Simultaneous imaging at several depths}

There is an unexploited potential in multiplexing $e F$-OCT images: this refers to the possibility of improving the overall number of Mvoxel/s that can be acquired in TD-OCT by parallel data acquisition. The time to investigate the tissue in volume using $e F-O C T$ could be reduced using a multiple channel configuration, where each channel selects a C-scan from a different depth. Several possible configurations have been investigated by the $\mathrm{AOG}$ to acquire a $N_{z}$ number of C-scans simultaneously, each of $N_{x}, N_{y}$ pixels, from $N_{z}$ different depth positions.

\subsubsection{Unbalanced multi-interferometer OCT configuration}

In a first attempt, two en-face images from the retina of a post mortem human eye were collected from different depths [34]. Such a setup is shown in Fig. 8. Two Michelson interferometers are used where frequency shifting at different carrier frequencies is imprinted, by using mirrors $\mathrm{M}_{1}$ and $\mathrm{M}_{2}$ that are vibrated by two electrostrictive elements $\mathrm{EE}$, driven by sinusoidal generators $\mathrm{G}_{1}$ and $\mathrm{G}_{2}$, at $f_{1}=30 \mathrm{kHz}$ and $f_{2}=22.5 \mathrm{kHz}$ respectively. A difference between the depths of two interferometers was chosen $1 / 4$ from the axial range investigated, i.e. $250 \mu \mathrm{m}$. This was adjusted by shifting the supports of the fiber ends and collimators $C_{1}, C_{2}$ relative to mirrors $\mathrm{M}_{1}$ and $\mathrm{M}_{2}$. Two photodetectors, $\mathrm{PD}_{1}$ and $\mathrm{PD}_{2}$, collect the returned optical signals. After photodetection, the signals in two channels are band pass filtered
(BPF), at $2 f_{1}\left(2 f_{2}\right)$ in order to avoid the residual intensity modulation on the fundamental frequency.

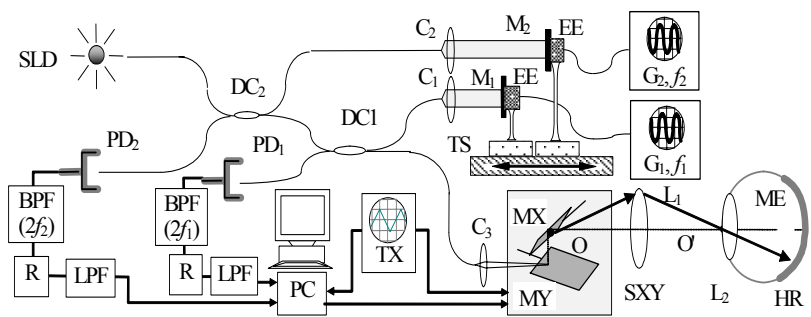

Fig. 8 Schematic diagram for en-face OCT at two different depths simultaneously (SLD: superluminescent diode $(850 \mathrm{~nm})$; $\mathrm{C}_{1}, \mathrm{C}_{2}, \mathrm{C}_{3}$ : microscope objectives; $\mathrm{DC}_{1}, \mathrm{DC}_{2}$ : directional couplers; $\mathrm{M}_{1}, \mathrm{M}_{2}$ : mirrors; $\mathrm{PD}_{1}, \mathrm{PD}_{2}$ : photodetectors; $\mathrm{SXY}$ : orthogonal galvo scanners; MX(MY): mirror of the $X(Y)$ scanner; ME: model eye consisting of a lens, $\mathrm{L}_{2}$ and human post mortem retina, HR; BPF: bandpass filter; R: rectifier; LPF: low pass filters; TS: computer controlled translation stage; EE: electrostrictive element; $\mathrm{G}_{1}, \mathrm{G}_{2}$; sinusoidal generators).

The signals are then rectified (R) and low pass filtered (LPF). The triangle generator TX drives the horizontal line scanner, MX, and triggers the acquisition of the two analog signals via an analog-to-digital (A/D) interface. Data acquisition and hardware commands are synchronised under the control of a LabView ${ }^{\mathrm{TM}}$ virtual instrument (VI). This VI also produces incremental voltage steps via a digital-to-analog (D/A) interface to drive the vertical scanner, MY. With MX driven at $20 \mathrm{~Hz}$, peak to peak amplitude $1 \mathrm{~V}$, and MY driven over 100 steps from $-0.5 \mathrm{~V}$ to $0.5 \mathrm{~V}$, the images in Fig. 9 were obtained. It can be noticed that the upper image in the set for $z=250 \mu \mathrm{m}$ becomes similar to the lower image in the set for $z=0 \mu \mathrm{m}$. Due to the low frequency phase modulation and scanning rate used, the display of the pairs of images required $3 \mathrm{~s}$. This method could be extended to display more than two layers in depth. An increase in the number of layers should be accompanied by a corresponding increase in the optical source power to compensate for the added losses. It can be noticed that the useful interference term stays the same irrespective of the number of interferometers used if the source power is correspondingly increased. The diagram in Fig. 10 shows the concept extended to 4 interferometers. 


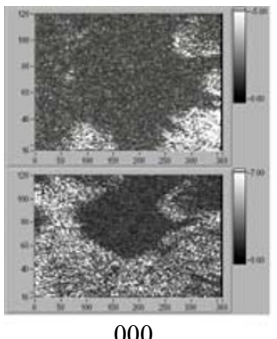

000

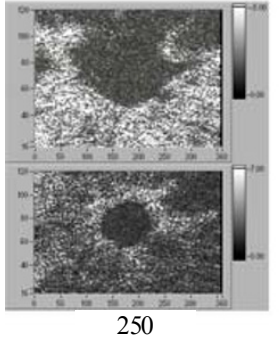

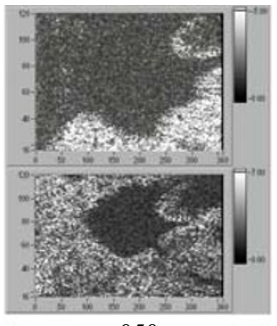

050

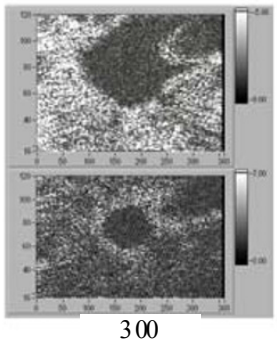

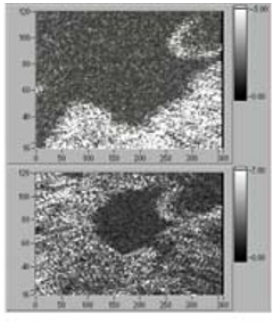

100

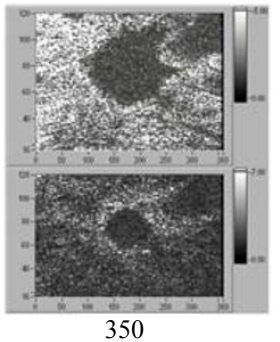

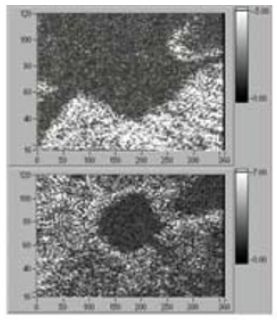

150

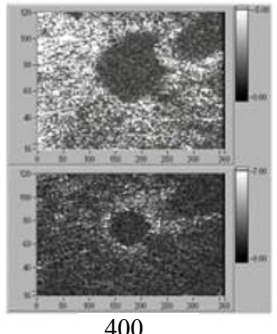

400

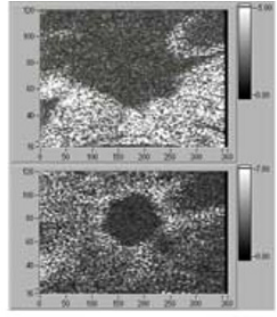

200

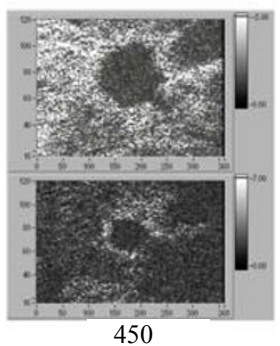

Fig. 912 pairs of $4 \mathrm{~mm} \times 4 \mathrm{~mm}$ C-scan OCT images taken at $50 \mu \mathrm{m}$ intervals from an in vitro retina tissue.

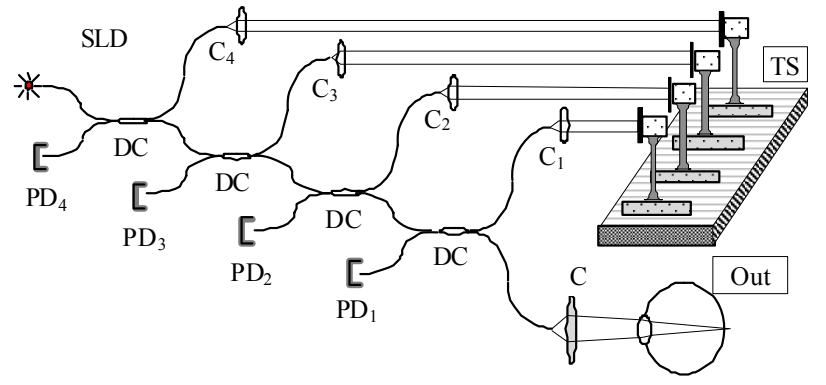

Fig. 10 System with 4 couplers (interferometers) for the simultaneous display of 4 C-scan OCT images (SLD: superluminiscent diode; DC: single mode directional couplers; $\mathrm{PD}_{1}-\mathrm{PD}_{4}$ : photodetectors; $\mathrm{TS}$ : computer controllable translation stage; $\mathrm{C}, \mathrm{C}_{1}-\mathrm{C}_{4}$ : microscope objectives; OUT: object under test).

An important constraint on increasing the number of layers (and accordingly of interferometers) derives from the fact that the interferometers are not physically independent. The interference signal from the first interferometer is present on the photodetector in the second interferometer, and the interference signal from the second is present in the third interferometer and so on. The electronics in the $n$th interferometer has to filter out $n-1$ interference signals. Therefore, an improved concept was developed as shown in the next paragraph.

\subsubsection{Simultaneous C-scan imaging using a balanced OCT configuration}

Simultaneous C-scan OCT imaging at multiple depths and rejection of excess photon noise are possible using an integrated modulator. A configuration with two channels [35] is shown in Fig. 11. The scheme employs only one interferometer with radio frequency multiplexing-demultiplexing.

The configuration is based on a single mode coupler array. Light from a pigtailed superluminescent diode, SLD is injected into a single mode coupler, DC1. The SLD delivers $120 \mu \mathrm{W}$ to the object and has a central wavelength at $860 \mathrm{~nm}$ and a spectral FWHM of $18 \mathrm{~nm}$. Assuming a Gaussian spectral profile, the coherence length, $l_{\mathrm{c}}$ $=32 \mu \mathrm{m}$ gives a depth sampling interval of $16 \mu \mathrm{m}$ in air. In the object arm, the light propagates from the port $\mathrm{S}$ via a microscope objective $\mathrm{C}_{1}$, the galvanometer scanner head SXY, and passes through lens $\mathrm{L}_{1}$, focal length $6 \mathrm{~cm}$ to the object. Two saw-tooth generators, RX and RY, drive mirrors MX and MY of the SXY scanning head.

The transmitted light from the other port of $\mathrm{DC}_{1}$ (the reference beam), which is of much higher power than that in the object beam, is transferred via ferrule, F, to the integrated optic Mach-Zehnder modulator (IOMZM). The ferrule $\mathrm{F}$ is in direct contact with the input guide of the IOMZM. The light at its output is collected via a microscope objective, $\mathrm{C}_{2}$, and then re-routed by mirrors $\mathrm{M}_{1}$ and 
$\mathrm{M}_{2}$ to the second coupler $\mathrm{DC}_{2}$. Two IOMZM electrodes are driven by sinusoidal signals from two generators $\mathrm{G}_{1}$ and $\mathrm{G}_{2}$ at $f_{1}=1.5 \mathrm{MHz}$ and $f_{2}=5 \mathrm{MHz}$ respectively. The mirrors serving to re-circulate the light in the reference arm are mounted on a computer controlled translation stage TS to enable coherence matching of the reference and the object arm. Polarization controllers, $\mathrm{FPC}_{1}, \mathrm{FPC}_{2}$, are mounted in the object and in the reference arms. Two photodetectors, $\mathrm{PD}_{1}$ and $\mathrm{PD}_{2}$, collect the returned optical signals from the coupler $\mathrm{DC}_{2}$. The photodetected signals are applied to two inputs of a differential amplifier, DA, in a balanced detection configuration. After DA, the signal is split into two electronic channels, each equipped with a notch filter, $\mathrm{NF}_{1}$ and $\mathrm{NF}_{2}$, and band pass filters, $\mathrm{BPF}_{1}$ and $\mathrm{BPF}_{2}$, a rectifier, $\mathrm{R}$, and a low pass filter, LPF. The system has consequently two channels, providing two OCT images, which are displayed simultaneously.

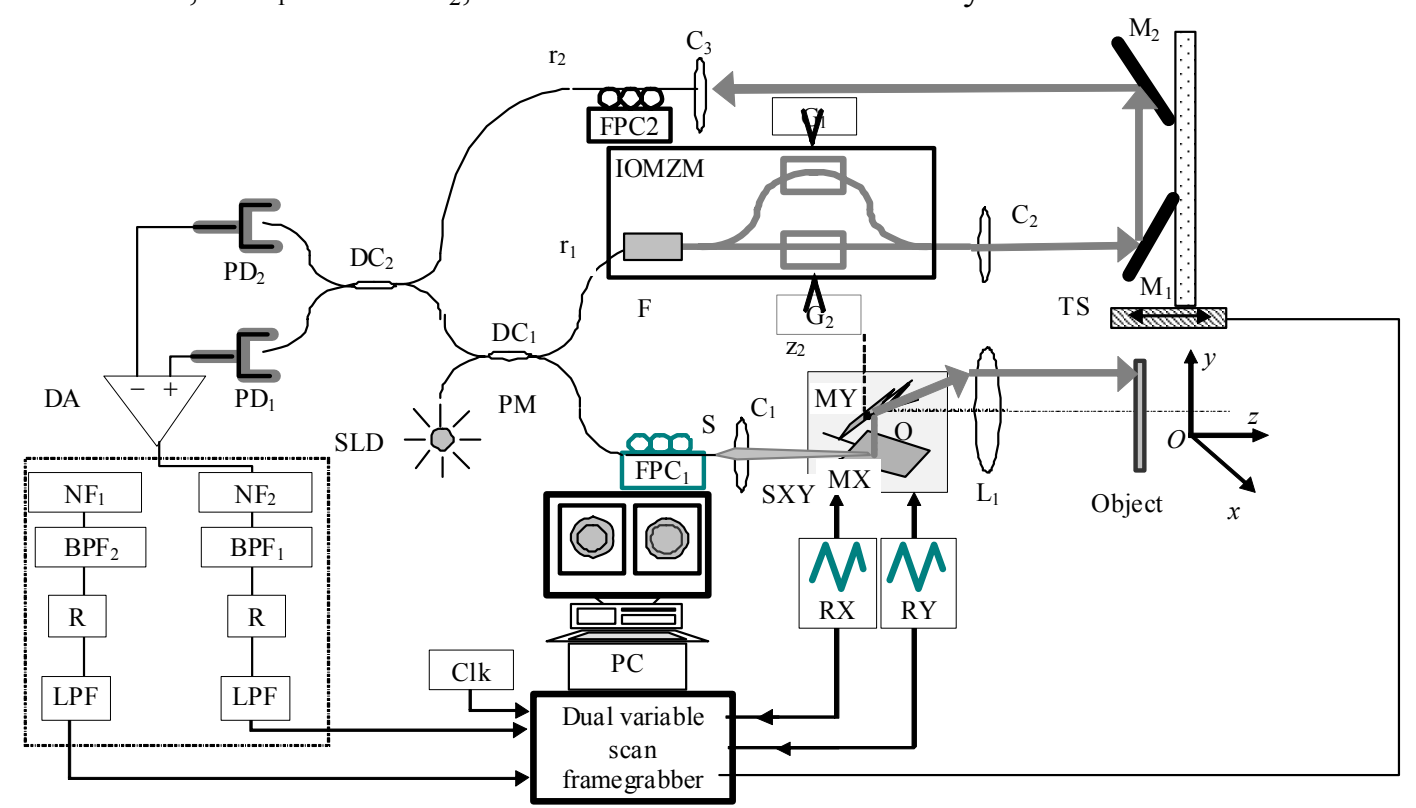

Fig. 11 Experimental setup for simultaneous $e F$-OCT imaging at two depths.

Saw-tooth signals of $700 \mathrm{~Hz}$ and of $2 \mathrm{~Hz}$ are applied to the $X$ - and $Y$-galvanometer scanners respectively. For an image size of $150 \times 150$ pixels, the image bandwidth required is about $100 \mathrm{kHz}$.

Modulating only one electrode at a time and processing the signal received from a mirror used as a target on the corresponding frequency, the correlation profile shown in Fig. 12 was obtained. Channel 2 uses the straight guide (undelayed) and exhibits a FWHM sectioning interval of $40 \mu \mathrm{m}$. Channel 1 uses the bent waveguide (delayed) and exhibits a FWHM sectioning interval of $120 \mu \mathrm{m}$. The sectioning intervals in both channels larger than the value of $16 \mu \mathrm{m}$ (given by half of the coherence length $l_{c}$ ) show dispersion in the system with a larger dispersion component in the bent waveguide channel.

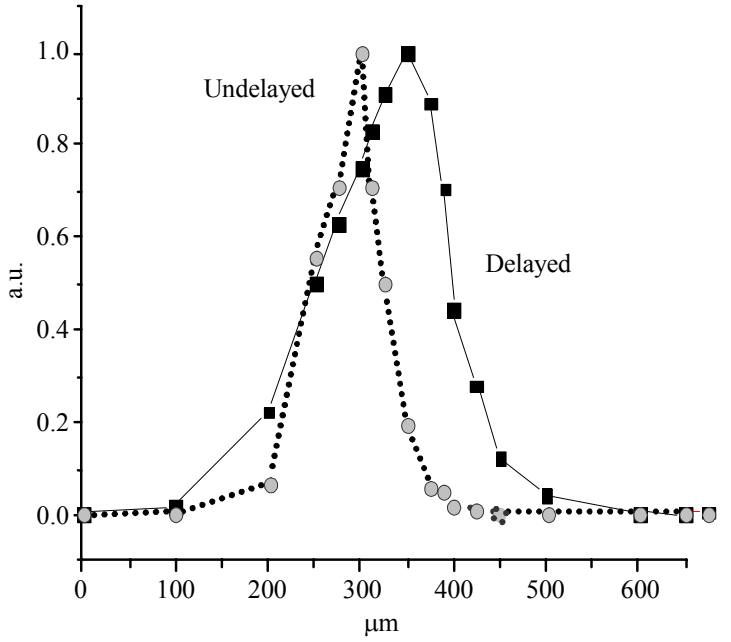

Fig. 12 Depth sectioning interval of two channels measured using a mirror as object, normally oriented to the system axis $Z$.

Simultaneous $e F$-OCT imaging at two depths is illustrated using a 5-pence coin as object. The 
amplitude on the two galvanometer scanners was such as to cover an area of $3 \mathrm{~mm} \times 3 \mathrm{~mm}$ at the back of the lens $\mathrm{L}_{1}$. Both BPFs were set at pass bands of about $100 \mathrm{kHz}$ and the low pass filter cut-off was adjusted to about $150 \mathrm{kHz}$.

The coin is an example of a two layer object in depth separated by about $60 \mu \mathrm{m}$, one layer, the plane of the coin background and the second layer, the top of the embossed letters. In channel 1 (Fig. 13 bottom), the background around the letter $\mathrm{R}$ is displayed while in channel 2 (Fig. 13 top), the peaks of the letter $\mathrm{R}$ and some of the areas surrounding the letter $G$ are displayed, showing that the coin is inclined with respect to the $O Z$ axis. The presence of dispersion in the curved waveguide affects the achievable performance of the system. If more channels are needed and longer waveguides need to be produced, these will exhibit even larger dispersion. Therefore, the design of a better modulator with less dispersion depends on the progress in the technology of integrated optic modulators.

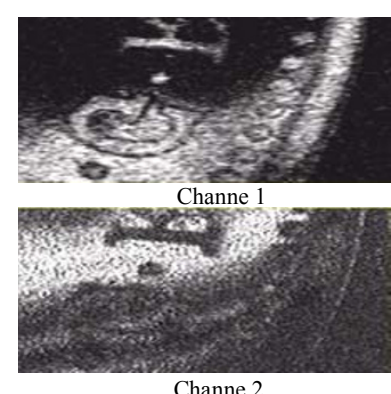

Fig. 13 Simultaneous en-face OCT imaging at two depths from a 5-pence coin, using the setup in Fig. 12 (transversal size: $3 \mathrm{~mm} \times 3 \mathrm{~mm})$.

\subsubsection{Multiple path interferometer configuration}

The solutions previously studied exhibited increased losses with the number of channels [35] or mismatched dispersion in multiple path modulators [36]. They limit the number of channels to a reduced number and the waveguide modulator solution is not reconfigurable.

A novel configuration was reported by the AOG recently, which allows interrogation of multiple depths separated by an interval which could be easily adjustable from zero to values less, larger or much larger than the coherence length of the optical source. Key to the new configuration presented is the use of re-circulating loops in both reference and sample arms of a low coherence interferometer, as shown in Fig. 14. Light from a broadband source, BBS, central wavelength $1060 \mathrm{~nm}$, full width at half maximum $50 \mathrm{~nm}$ (Multiwave Photonics, Porto, Portugal), is split into a main sample arm and a main reference arm by the directional coupler, DC. Each main arm of the interferometer incorporates an acousto-optic frequency shifter, $\mathrm{AOFS}_{1}\left(\mathrm{AOFS}_{2}\right)$, which shifts the optical frequency upwards by the frequency of the driving signal $f_{1}\left(f_{2}\right)$. The setup is based on a Mach-Zehnder interferometer, where each main arm feeds a separate optical ring, with adjustable path length. To compensate for the losses, identical semiconductor optical amplifiers, $\mathrm{SOA}_{1}$ and $\mathrm{SOA}_{2}$ (QPhotonics, QSOA-1050) are incorporated in two rings. In this way multiple depths can be interrogated, separated by the difference between the optical path lengths of two rings, OPLDiff. The multiple delays thus introduced are encoded on the RF frequency given by the beating of $f_{1}$ and $f_{2}$. After the $\mathrm{AOFS}_{1}\left(\mathrm{AOFS}_{2}\right)$, light is injected into the main loop as well as into a sample (reference) ring via a coupler $\mathrm{DC}_{1 \mathrm{~b}}\left(\mathrm{DC}_{2 \mathrm{~b}}\right)$. Optical isolators $\mathrm{I}_{1 \mathrm{a}}, \mathrm{I}_{1 \mathrm{~b}}\left(\mathrm{I}_{2 \mathrm{a}}, \mathrm{I}_{2 \mathrm{~b}}\right)$ placed at the input and at the output of the $\mathrm{SOA}_{1}\left(\mathrm{SOA}_{2}\right)$ protect their operation from stray reflections. The amplified light is re-injected into the main loop by a coupler $\mathrm{DC}_{1 \mathrm{a}}$ $\left(D_{2 \mathrm{a}}\right)$. For every pass of light through the sample (reference) ring, the optical frequency is shifted, by $f_{1}\left(f_{2}\right)$. The main sample arm contains an optical circulator $\mathrm{C}$, microscope objective $\mathrm{MO}_{1}$, a pair of orthogonal galvo-scanners SXY, and a lens $\mathrm{L}_{1}$, sending $1 \mathrm{~mW}$ towards the sample. Its focal length of $2 \mathrm{~cm}$ and the $\mathrm{MO}_{1} \times 10$ determine a spot size on the sample of $10 \mu \mathrm{m}$. The multiple waves from the reference path interfere with the multiple waves from the sample path at the directional balanced 
coupler $\mathrm{BC}$, producing a beat $f=n\left|f_{1}-f_{2}\right|=n \Delta f$, where $n$ is the number of re-circulations of light in two rings. The $\mathrm{BC}$ output signals are sent to a balanced photo-detector unit, consisting of two pin photo-detectors, $\mathrm{PD}_{1}, \mathrm{PD}_{2}$, and a differential amplifier, DA. In this way, the OCT signal collected by en-face imaging of the target is placed around a carrier of frequency $n \Delta f$. The AOFS ${ }_{1}$ is driven at $f_{1}=$ $40 \mathrm{MHz}$ whilst $\mathrm{AOFS}_{2}$ is driven from a generator which allows tuning of $f_{2}$ to adjust $\Delta f$. For a $500 \mathrm{~Hz}$ line rate, the bandwidth of the OCT signal is in the range of hundreds of $\mathrm{kHz}$. Therefore, $f_{2}$ is chosen 41 $\mathrm{MHz}$ to determine a carrier frequency $\Delta f=1 \mathrm{MHz}$.

The number of re-circulation passes through the rings determines the number of $e F$-OCT images that can be collected for different depth positions of the coherence gate, at intervals determined by OPLDiff measured from the depth in the sample where the main OPD is zero.

The optical path length in the rings can be adjusted using the micrometer screws of the translation stages, TS, that allow axial movement of microscope objectives, $\mathrm{MO}_{4}-\mathrm{MO}_{7}$, and fiber receptacles together.

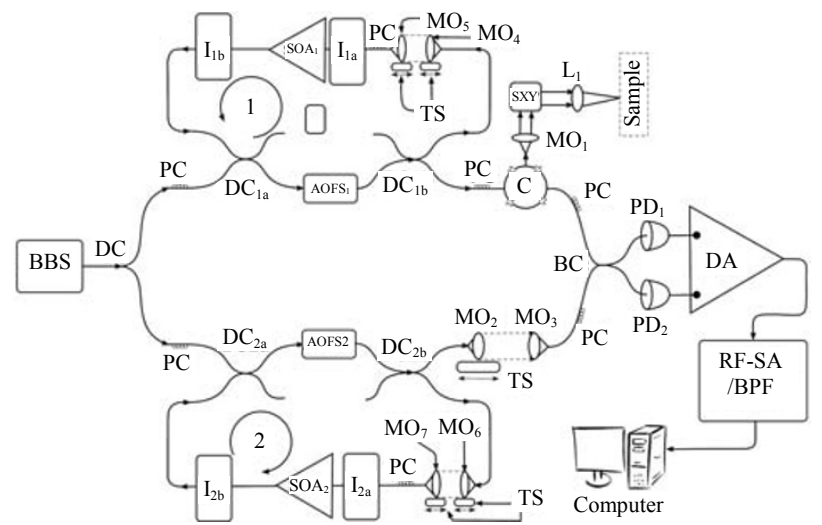

Fig. 14 Schematic diagram of the multiple depths $e F$-OCT system.

To prove such capability, the system was used to simultaneously acquire images from a Drosophila in its 3rd larval instar stage prepared as described in [34]. Five such eF-OCT images are shown in Fig. 15. The OPLDiff was set on $50 \mu \mathrm{m}$. In this way multiple interference signals were produced via multiple re-circulations at progressive $50 \mu \mathrm{m}$ steps in depth in the fly. Images of $2.1 \mathrm{~mm}$ (horizontal) and $1.1 \mathrm{~mm}$ (vertical) in size were obtained by driving two galvo-scanners with ramps at $500 \mathrm{~Hz}$ and $2 \mathrm{~Hz}$ respectively (each image of 500 lines).

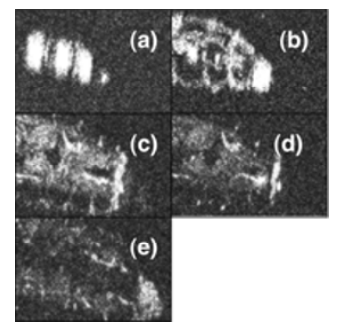

Fig. 15 Simultaneously generated $e F$-OCT images of a Drosophila larva with its dorsal side up (posterior to the right) from 5 different depths, selected when the RF-SA was tuned on $f=1 \mathrm{MHz}(\mathrm{a}), 2 \mathrm{MHz}$ (b), $3 \mathrm{MHz}$ (c), $4 \mathrm{MHz}$ (d), and $5 \mathrm{MHz}(\mathrm{e})$.

\subsubsection{Multiple delay element in the reference arm}

We have been exploring the possibility of reducing the time required for measurement of the cornea shape by taking multiple transversal scans at different depths at the same time. This application is different from the task in previous paragraphs, of collecting C-scan images at different depths. Here we are interested in the topography of single layer objects only and a simplified method is proposed, where all C-scans at several depths are superposed together. To this goal, a reference path composed of multiple optical path lengths is used. This is achieved by introduction of a multiple delay element (MDE) in the OCT reference arm [36]. A MDE is constructed from stacked thin microscope glass slides. We will refer to an MDE equipped OCT system as an MDE-OCT. An MDE-OCT C-Scan image is obtained in the time normally required to obtain a single $\mathrm{C}$-scan, yet it contains the information needed to determine three dimensional shape of curved objects, with applications in topography of the cornea. Since the measurement time is reduced in comparison with either scanning at multiple depths in case of a C-scan based OCT system or at different polar orientations in case of a B-scan based OCT system, the result is less affected by eye movement. Using this method, the axial 
position of or the radius of a spherical object can be measured.

The schematic diagram of the $e F$-MDE-OCT setup is shown in Fig. 16. The system employs a two-coupler configuration and the source is a 1300-nm super-luminescent diode sending 1-mW optical power towards the cornea. The depth resolution of the system is $18 \mu \mathrm{m}$ in air. A low numerical aperture is used, by employing an achromat lens of 4-cm focal length between the $X Y$ scanning block and the cornea. A galvanometric optical scanner pair are used to transversally scan the beam across the cornea in the transverse plane $(X$, $Y)$. The $X$-scanner is driven at $500 \mathrm{~Hz}$ and the $Y$-scanner is driven at $2 \mathrm{~Hz}$. The axial position of the scan is controlled by moving the reference mirror, which is mounted on a $1-\mu \mathrm{m}$-precision translation stage, TS. The reference beam intersects the MDE, which generates multiple optical path delays. A combined C-scan image obtained from this system is the superposition of the C-scan OCT images which could be obtained for each and every optical path in the reference arm. The combined C-scan OCT image can be used to generate the cornea topography, as the height of the cornea shape is coded in the radius of contours corresponding to the interface between air and the cornea. The acquired multiple contours $\mathrm{C}$-scan is measured and analyzed by a computer program, identifying cornea surface locations that are located at a specific axial distance, which can be used to produce a three dimensional cornea topography dataset.

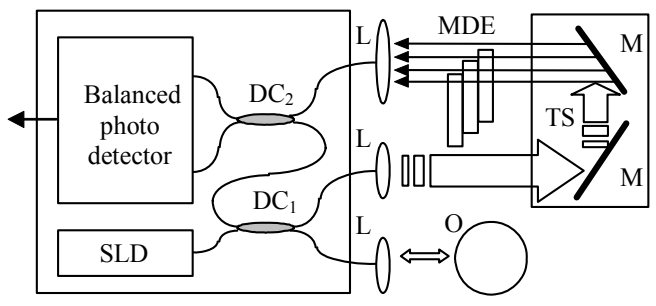

Fig. 16 Diagram of the multiple delay OCT system (SLD: super-luminescent diode; $\mathrm{DC}_{1}, \mathrm{DC}_{2}$ : single mode directional couplers; L: lenses; M: mirrors; MDE: multiple delay element with 3 layers, which introduces 4 different length reference paths; TS: translation stage; O: object to be measured).
Topography of an eye obtained with an MDE with 5 plates is shown in Fig. 17.

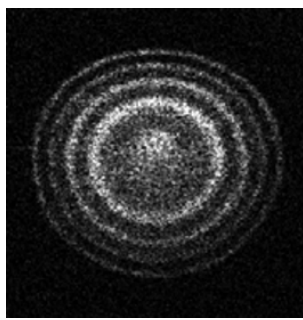

(a)

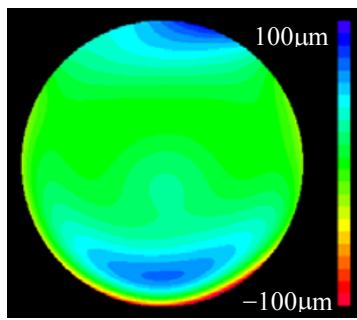

(b)
Fig. 17 (a) MDE-OCT C-Scan image of the cornea of a volunteer and (b) cornea elevation map obtained from the image at (a)).

The method can be extended to measurement of topography of any single layer reflective object. In the time required to produce one combined $\mathrm{C}$-scan frame (the same as the time required for a single C-scan), all contours are obtained.

The same principle was also tested using a full field OCT setup and a 2D camera [37] to measure the curvature of a metal ball in one shot.

\subsection{Other contributions to TD-OCT}

Several other directions have been approached to strengthen the TD-OCT technology. In order to simplify the optics of OCT/SLO and OCT/confocal configurations by eliminating the splitter used to sample a fraction from the signal returned from the object to serve the SLO (confocal) channel, sequential mode of operation was proposed [38] or quasi-simultaneous, during half periods of the transversal scanning period [39]. Sequential and quasi-simultaneous eF-OCT/SLO imaging was proven with images collected from the eye in vivo.

A way forward to ease the interpretation of C-scans from curved tissue, which look fragmented, is to increase the coherence length of the optical source, or employ superposition of several neighboring C-scans. In this way, $e F$-OCT with adjustable depth range [40] is achievable.

In order to improve the efficiency of spectral scanning delay lines, which normally use 4 passes via the dispersing element, a configuration was proposed and studied with only two passes. In 
addition, the configuration suggested operates in transmission, which makes it compatible with balance detection schemes using recirculation of the reference beam [41].

\section{AOG contributions to SD-OCT}

SD methods can be implemented in two formats: 1) by spectrally dispersing the channelled spectrum (CS) at the interferometer output (often referred to as Fourier domain OCT) and 2) by using a tunable laser or a swept source (SS). Each method, CS-OCT or SS-OCT, has its own merits and deficiencies.

Between 1990 and 1995, research was ongoing within the AOG on measuring the small distance between the piston and the cylinder in a thermal engine, using a spectrometer to analyse the spectrum output from a Michelson interferometer formed between the fiber end and the piston [42]. This stage in research represents also an early example of a common path configuration evaluated by the AOG. The technology was first developed for LCI measurement [43] of thicknesses and indexes of refraction [44] and for an easy demonstrator of channeled spectrum for a Physics lab [45]. The experience acquired in these studies allowed AOG to quickly move to CS-OCT after 2004.

\subsection{Initial research on Talbot bands}

By 1993, in the process of assembling channeled spectrum setups, we stumbled upon the generation of Talbot bands. Talbot bands represent a curious effect, discovered by Talbot in 1837 . When a glass plate is introduced halfway into the beam coming from a white light source, modulation of the spectrum in the form of a CS or Talbot bands appear only when the glass plate intercepts a certain side of the beam. G. B. Airy dispelled any "curious" effects as mere results of interference which enhances the amplitude of some wavelengths and reduces the amplitude of others. When using a prism, the plate should be introduced into the side corresponding to the red part of the dispersed spectrum while when using a diffraction grating, the plate should be introduced into the blue side of the beam.

In a theoretical [46] and experimental [47] report, we demonstrated that a modified Michelson configuration could be used for studying Talbot bands. For the case of a laser diode below threshold used as a low coherence source, by introducing screens in a Michelson interferometer, the fast Fourier transform (FFT) spectrum of the CS obtained was simplified. In these reports we have shown that the CS contains components due to the combination of the OPD in the interferometer with the OPD equivalent to the laser cavity length. The simplification of the FFT spectrum of the CS is intimately related to the process of elimination of mirror terms. CS bands are visible for one sign of the OPD only and there is an optimum OPD where the visibility is maximum. These particularities are characteristics of Talbot bands and they make Talbot bands ideal for elimination of mirror terms.

The problem of mirror terms in OCT is equivalent to the problem of OPD sign ambiguity in sensing. SD-OCT image from a real-valued spectral interferogram consists of two overlapped images that are symmetrical with respect to the zero plane (zero-phase delay) of the interferometer. The zero plane must be positioned outside the imaged sample which reduces the imaging depth range to less than half. Mirror terms are responsible for ghost images in SD-OCT. The same image (upside down) results for the same modulus value of the OPD.

\subsection{Talbot bands in optical coherence tomography}

Once realization of the potential of Talbot bands for OCT is clear, theoretical and experimental directions have been investigated for the adaptation of configurations known to exhibit Talbot bands in performing OCT.

\subsubsection{Theoretical studies}

Two theoretical models have completed this line of research so far. A first report provides a unique 
interpretation for Talbot bands, spectral domain white light interferometry and FD-OCT setups [48]. A second report [49] demonstrates a formula for the visibility of FD-OCT due to the Talbot bands.

\subsubsection{Experimental studies}

A first experimental setup has demonstrated that indeed, B-scan OCT images totally deprived of mirror terms could be obtained [50]. The method was proven in collecting OCT images free of mirror terms from skin. The first setup had the disadvantage of sending some light from the reference to the optical source. A second experimental report [51] improved on this aspect and the setup used is shown in Fig. 18. This has also provided a more complete description of the visibility decay with depth in any FD-OCT setup producing or not Talbot bands, given by

$$
V(\mathrm{OPD})=C_{\mathrm{OR}}\left(\frac{\sin (\xi)}{\xi}\right)^{2}
$$

where $\xi=(\pi / 2) \cdot\left(\mathrm{OPD} / z_{\mathrm{RD}}\right)$ is the depth normalised by the first sinc zero, $z_{\mathrm{RD}}=\lambda^{2} /(4 \Delta \lambda)$ is the central wavelength, and $\lambda$ is the mean wavelength range which satisfies the grating equation for individual pixels. The second factor, subsequently referred to as the sinc term, describes attenuation due to the finite size of the photo-detectors in the spectrometer.

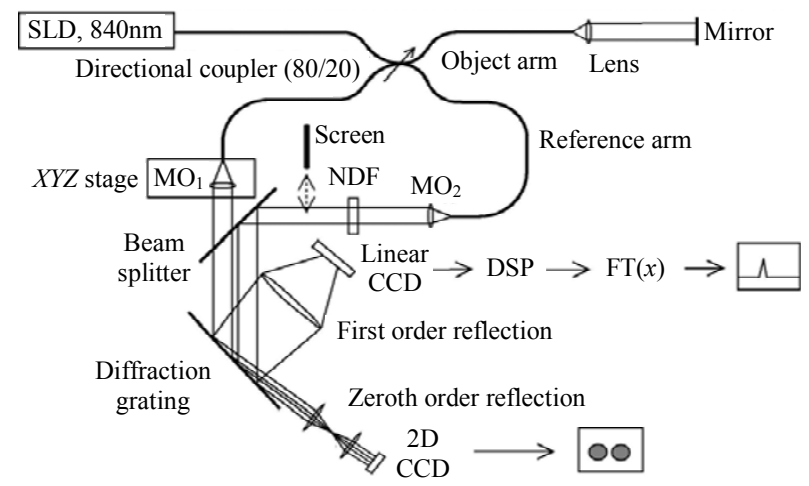

Fig. 18 Talbot bands OCT setup (NDF: neutral density filter; $\mathrm{MO}_{1}$ : object beam microscope objective; $\mathrm{MO}_{2}$ : reference beam microscope objective; FT( $(x)$ : 1D Fourier transform).

The first factor, $C_{\mathrm{OR}}$ is given by the correlation of two functions, $B_{\mathrm{O}}$ and $B_{\mathrm{R}}$, which describe the field strength spatial profile at the grating within the object and reference beams respectively. In conventional FD-OCT. $B_{\mathrm{O}}$ and $B_{\mathrm{R}}$ are described by Gaussians and the resultant correlation, $C_{\mathrm{OR}}$, provides a Gaussian component of the visibility fall-off, as predicted by [50]. We have shown that the sensitivity decay, $V(\mathrm{OPD})$, can be explained by considering the amount of overlap, $C_{\mathrm{OR}}$, of the object and reference beam wavetrains after diffraction. The models in [48] and [49] are more general than the explanation in reports where the sensitivity decay is justified by considering the resolving power of the spectrometer optics only. Specifically, our model provides for cases where the diffraction grating is illumined by non-super-imposed beams, resulting in visibility profiles which could continue to be Gaussian, but could also be non-Gaussian or have a peak at OPD values away from zero. We have shown in [49] that for the case when the two beams are superposed on the grating $B_{\mathrm{O}}$ and $B_{\mathrm{R}}$ have Gaussian spatial profiles and $C_{\mathrm{OR}}$ exhibits a Gaussian profile as well.

\subsubsection{Distinction between using Talbot bands and other approaches to reduce the strength of mirror terms}

All other known methods to reduce the mirror terms are based on cancellation techniques (and so require good stability of parameters and are sensitive to movement, which affect the equilibrium of quantities which are added up to cancel the mirror terms) requiring several images or steps. Such a method is superior to all other known methods to resolve the ambiguity in the OPD sign, all based on cancellation techniques inspired from phase shifting interferometry or complex Fourier signal processing. The prior art methods require several steps acquisitions, calculations which take time and also well adjusted and stable parameters. The Talbot bands method does not require combination of signals and computation, and simply a Talbot bands LCI is sensitive to one sign of the OPD only. Using Talbot bands, the mirror terms practically do not exist, so such method eliminates the OPD sign 
ambiguity in one step (critically important for moving organs).

\section{Trends in OCT imaging applications}

More than $50 \%$ of published reports on OCT worldwide are within subjects of ophthalmology and optometry. The orientation of projects within the AOG also reflects this trend, with majority of research on imaging the eye. In this respect, in addition to the work presented above on the OCT/SLO and OCT/ICG, two other major trends were achieving high depth resolution and operating the same concepts of OCT/SLO at longer wavelengths. Using conventional TD-OCT, based on A-scans, it was proven that from the eye in vivo, 3-micron depth resolution could be achieved. In collaboration with New York Eye and Ear Infirmary and OTI Toronto Canada, we embarked on a project aiming to establish the axial resolution limit in using $e F$-OCT. Ultra high resolution images of the retina have been obtained with the OCT/SLO [52], where the $\mathrm{C}$-scan slice thickness and resolution in the B-scan OCT images reached the same value of 3 microns, reported before.

In order to penetrate deeper into the choroid, the OCT/SLO project was extended to $1050 \mathrm{~nm}$, where the scattering is less than that at $800 \mathrm{~nm}$ and there is an absorption dip in the curve of water absorption [53].

Another trend is that of extending the technology developed initially for the eye imaging to other medical imaging applications. These include 3D imaging of skin [54], in vitro imaging of basal cell carcinoma [55], in vitro imaging of larynx tissue [56], and studies of demineralization of teeth [57]. Applications in biology have also been targeted such as imaging of Drosophila larvae heart [33] and imaging of collagen based constructs [58].

A particular application direction is that of assessing the capability of OCT for art conservation. We have proven that OCT can be used to monitor the thickness of varnish layers during conservation work of painting and that the $e F-\mathrm{OCT}$ can detect underdrawings better than an InGaAs camera [59]. In addition, recently AOG has demonstrated that a specialized probe head in tandem with a fast OCT method (such as a SS-OCT) can provide speckle free images [60].

\section{Novel scanning configurations}

\subsection{Spectral scanning delay line}

A lower loss transmissive grating-based scanning spectral delay line was proposed [61]. This is especially suited to fit recirculating paths in OCT balanced configurations. The walk-off could be de-scanned with only two passes via the grating, instead of four in the reflecting spectral scanning delay lines.

\subsection{Scanning head for speckle averaging}

We proposed a sequential angular compounding method for reducing speckle contrast in OCT images [60]. A galvanometer scanning head was mounted on a motorised translation stage, allowing translation perpendicular to the optical axis. Such a translation alters the angle of incidence of the sample beam on the target but does not change its transversal position, or the optical path length, providing the target is at focus. The advantage of this method is that the maximum angle obtainable is not dependent on the size of the galvanometer mirror, and depends ultimately only on the diameter of the sample lens. In addition, the method can be extended to produce de-speckled cubes.

This method permits a larger variation in the angle of incidence as well as the de-speckling of cubes.

The results were compared with post-processing methods and showed that the compounding technique improved the speckle contrast ratio in B-scans by better than a factor of 2 in exchange for a negligible loss of resolution. As a result, OCT image aesthetics are improved, and thin layers become more distinct and edge-detection algorithms work 
more efficiently. So far, the method was applied for imaging objects of art and teeth in vitro.

\subsection{Dynamic focus}

A simplified method for dynamic focus was proposed where the same scanner performed coherence gate axial tuning and focus adjustment at the same time [62]. By allowing physical separation of two interferometer arms, the AOG method removes a design constraint which restricts the layout of more complex systems such as those employing adaptive optics. In addition, it permits the design of a compact probe-head which can be positioned independently of the rest of the OCT system. This allows for much greater flexibility in creating systems which are portable and which can be employed in a wide range of situations. The method ensures synchronism of the confocal and coherence gate for objects of index of refraction about 1.41 .

\subsection{Eye tracking}

Involuntary eye and head motion of patients during eye fundus imaging examination lowers the quality of the retinal scans due to intra-frame and inter-frame distortion. It also affects quantitative measurement performed by means of OCT. An active tracking device was developed based on a spectrally interrogated Michelson interferometer. This was used to monitor and correct for the axial displacement of the eye and head of the subject in an OCT/SLO system. The tracking and imaging interferometers share the eye interface optics as well as a fast voice coil mounted retro-reflector, that keeps the imaging interferometer locked at constant optical path difference irrespective of the axial eye movements. A real time data acquisition processor board is used to digitize the spectrometer signal and calculate the correction signal applied to the voice coil with an update time better than $5 \mathrm{~ms}$. We demonstrated $e F$-OCT frames corrected for axial motion, paired with SLO images of the eye fundus in vivo [63].

\section{Signal processing and signal to noise analysis}

An initial report has shown that in order to achieve fast acquisition, TD-OCT systems need to operate in excess photon noise regime using balance detection [64]. These results were then applied to a combined OCT/SLO system [65]. In a subsequent report, new noise bandwidth definitions were introduced to account for the spectral dependence of splitting coefficients of optical splitters [66]. In the same direction, the time variant second order statistics of the depth-scan photocurrent in TD-OCT systems using polarized thermal light sources was evaluated [67]. A study on what limits the sensitivity in a TD-OCT has led interestingly to a formula depending on the speed of light in the substance [68] and the background of multiply scattered light.

\section{AOG contributions to adaptive optics (AO) and wavefront sensing}

\subsection{AO assisted OCT, AO assisted microscopy}

Using a deformable mirror in the system in Fig. 6 , incorporated in a closed loop controlled by a Shack-Hartmann wavefront sensor, aberrations are reduced and a higher intensity signal is received at the photodetector and the transversal resolution in both images is improved [69]. OCT and SLO images acquired under AO control loop are presented in Fig. 19.
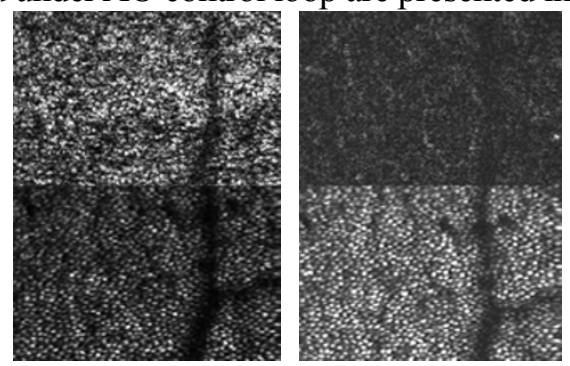

Fig. 19 Pairs of C-scan OCT and SLO images simultaneously acquired and displayed via AO closed loop correction in an OCT/SLO system (each image size: $330 \mu \mathrm{m}$ $\times 500 \mu \mathrm{m}$; top: OCT C-scan image; bottom: SLO image; the depth in the OCT channel is at the junction of inner segment and outer segment (left) and approx. $50 \mu \mathrm{m}$ above (right), displaying microvasculature). 


\subsection{Coherence gated wavefront sensor}

\subsubsection{Insensitivity to depth variations of aberrations of a standard Shack-Hartmann wavefront sensor}

Due to the limited numerical aperture in the beams associated with each microlens in the lenslet array, a Shack-Hartmann wavefront sensor has little sensitivity to the position in depth in the object where the signal comes from. This makes the Shack-Hartmann wavefront sensor insensitive to depth variations of aberrations, or more precisely, the spots are deviated from the ideal wavefront grid by quantities which represent averages of aberrations over the depth of focus of the equivalent confocal microscopy channel associated with each microlens in the lenslet array.

\subsubsection{Sensitivity to stray reflections of a standard Shack-Hartmann wavefront sensor (SH/WFS)}

Due to the insensitivity to depth mentioned above, stray reflections from the interface optics cannot be rejected and therefore supplementary spatial filters need to be employed in the interface optics. However, when imaging the eye, reflections from the cornea and the eye lens cannot be entirely attenuated using spatial filters.

\subsubsection{Coherence gating}

AOG has investigated the interference on a CCD array between multiple beamlet outputs by the lenslet array and a reference beam provided by the same optical source used to illuminate the target [70]. Due to the aberrations encountered by the beam towards and backwards from the target (in double pass configurations) or coming from the target only (in single pass configurations), the beamlets suffer various deflections on the CCD array. Irrespective of these deflections, interference with the reference beam still takes place, as the reference beam is uniformly spread over the CCD array. Two versions of coherence gated (CG) wavefront sensors were evaluated: 1) a time domain
(TD) version, driven by a source with large bandwidth and using principles of phase shifting interferometry and 2) a spectral domain version using a swept narrow band source (SS). The second possibility takes advantage of the recent advance in SS-OCT using a 2D camera and provides supplementary advantages such as dynamic spectral compensation for focus change. The two approaches are different in their outcome and utilization:

1) Using a broadband source, phase shifting interferometry is used to recover an en-face image of the spot pattern.

2) Under swept source illumination, A-scans are produced and therefore in order to generate an en-face image for the spot pattern, the whole volume of the tissue is first acquired and assembled from such multiple A-scans, volume which is then subsequently software cut perpendicularly to the direction of the A-scans.

Using both methods, it was proven that stray reflections in the optics could be successfully eliminated. This opens the possibility of using lenses in double pass configurations when imaging the retina and extending the SH/WFS principle to microscopy.

\section{Conclusions}

The work described here over the years is the result of leadership of Prof. David Jackson who successfully obtained research support for OFS from numerous sponsors. The orientation towards OCT is the result of an Engineering Physical Sciences Research Council grant initiated by Prof. David Jackson and lecturer David Webb. The subsequent successful OCT research is the result of many contributions from dedicated colleague researchers, such as postdoctoral researchers, Adrian Bradu, Mateias Bonmarin, Ramona Cernat, Radu Cucu, Mark Hathaway, John Rogers, lecturers George Dobre, Sherif Sherif, PhD students, such as Ismini Charalambous, Michael Hughes, Alexander Meadway, Dan Woods, Ryan Harding, Steve Taplin, 
Mauritius Seeger and Marie Curie researchers: Liviu Neagu, Erika Odlund, Florin Tudorache, Radek Base, Iwona Gorzynska, Marta Gomez, Boris Kudimov, Carla Rosa, Irina Trifanov, Simon Tuohy, Jingyu Wang.

Examples of old and recent research in the AOG in the field of fiber optic sensing, low coherence interferometry and OCT were presented. AOG has contributed towards TD and SD-OCT, towards simultaneous imaging at several depths, common path LCI and OCT configurations, optical sources for OCT and specific signal processing methods.

\section{Acknowledgment}

The results obtained would not have been possible without the support, contribution and encouragement of Prof. Fred Fitzke, from the Institute of Ophthalmology, UCL, London, Prof. Richard Rosen from the New York Eye and Ear Infirmary, Mr. Rishard Weitz, from Ophthalmic Technology Inc., and Prof. Bill Vennart from Pfizer, Sandwich.

Colleagues in the technical services, occupational office, research office and innovation and enterprise are acknowledged for their continued support in ensuring optimum conditions of work, education, and training.

Open Access This article is distributed under the terms of the Creative Commons Attribution License which permits any use, distribution, and reproduction in any medium, provided the original author(s) and source are credited.

\section{References}

[1] A. D. Kersey, D. A. Jackson, and M. Corke, "Passive compensation scheme suitable for use in the single-mode fiber interferometer," Electronics Letters, vol. 18, no. 9, pp. 392-393, 1982.

[2] A. Gh. Podoleanu, G. M. Dobre, D. J. Webb, and D. A. Jackson, "Coherence imaging by use of a Newton rings sampling function," Optics Letters, vol.
21, no. 21, pp. 1789-1791, 1996.

[3] A. Gh. Podoleanu, M. Seeger, G. M. Dobre, D. J. Webb, D. A. Jackson, and F. Fitzke, "Transversal and longitudinal images from the retina of the living eye using low coherence reflectometry," Journal of Biomedical Optics, vol. 3, no. 1, pp. 12-20, 1998.

[4] A. D. Kersey, M. Corke, and D. A. Jackson, "Linearized polarimetric optical fiber sensor using a heterodyne-type signal recovery scheme," Electronics Letters, vol. 20, no. 5, pp. 209-21, 1984.

[5] A. D. Kersey and D. A. Jackson, "Current sensing utilizing heterodyne-detection of the Faraday-effect in single-mode optical fiber," Journal of Lightwave Technology, vol. 4, no. 6, pp. 640-644, 1986.

[6] A. S. Gerges, F. Farahi, T. P. Newson, J. D. C. Jones, and D. A. Jackson, "Fiber-optic interferometric sensor utilizing low coherence length source-resolution enhancement," Electronics Letters, vol. 24, no. 8, pp. 472-474, 1988.

[7] S. C. Bartlett, F. Farahi, and D. A. Jackson, "Common-path optical fiber heterodyne interferometric current sensor," Proc. SPIE, vol. 1504, pp. 247-250, 1991.

[8] A. D. Kersey, D. A. Jackson, and M. A. Corke, "A simple fiber Fabry-Perot sensor," Optics Communications, vol. 45, no. 2, pp. 71-74, 1983.

[9] D. J. Webb, J. D. C.Jones, and D. A. Jackson, "Extended-range interferometry using a coherence-tuned, synthesized dual-wavelength technique with multimode fiber links," Electronics Letters, vol. 24, no. 18, pp. 1173-1175, 1988.

[10] Y. J. Rao, D. J. Webb, and D. A. Jackson, "Design study of fiber-optic based Fabry-Perot type interferometric sensors using low-coherence signal recovery," Proc. SPIE, vol. 2070, pp. 360-371, 1994.

[11] S. C. Bartlett, F. Farahi, and D. A. Jackson, "Current sensing using Faraday-rotation and a common path optical fiber heterodyne interferometer," Review of Scientific Instruments, vol. 61, no. 9, pp. 2433-2435, 1990.

[12] L. A. Ferreira, J. L. Santos, and F. Farahi, "Polarization insensitive fiber-optic white-light interferometry," Optics Communications, vol. 114, no. 5-6, pp. 386-392, 1995.

[13] G. P. Brady, K. Kalli, D. J. Webb, D. A. Jackson, L. Zhang, and I. Bennion, "Extended-range, low coherence dual wavelength interferometry using a superfluorescent fiber source and chirped fiber Bragg gratings," Optics Communications, vol. 134, no. 1-6, pp. 341-348, 1997.

[14] M. Corke, J. D. C. Jones, A. D. Kersey, and D. A. Jackson, "Two-dimensional laser Doppler velocimeter using polarization-maintaining single-mode fibers," in Optical Fiber Sensors, (Optical Society of America, 1985), paper 
ThGG6.vol. OFC/OFS 85', pp. 146, 1985.

[15] R. P. Tatam, C. N. Pannell, and J. D. C. Jones, "Full polarization state control utilizing linearly birefringent monomode optical fiber," Journal of Lightwave Technology, vol. 5, no. 7, pp. 980-985, 1987.

[16] D. A. Jackson, R. Priest, A. Dandridge, and A. B. Tveten, "Elimination of drift in a single-mode optical fiber interferometer using a piezoelectrically stretched coiled fiber," Applied Optics, vol. 19, no. 17, pp. 2926-2929, 1980.

[17] P. Merritt, R. P. Tatam, and D. A. Jackson, "Interferometric chromatic dispersion measurements on short lengths of monomode optical fiber," Journal of Lightwave Technology, vol. 7, no. 4, pp. 703-716, 1989.

[18] J. L. Santos and D. A. Jackson, "Time-division multiplexing exploiting low-coherence interferometry with a multimode laser diode source," Proc. SPIE, vol. 1586, pp. 184-193, 1992.

[19] A. S. Gerges, T. P. Newson, and D. A. Jackson, "Interferometric fiber-optic sensing using a multimode laser diode source," Proc. SPIE, vol. 1504, pp. 176-179, 1991.

[20] Y. J. Rao and D. A. Jackson, "Long-distance fiberoptic white-light displacement sensing system using a source-synthesizing technique," Electronics Letters, vol. 31, no. 4, pp. 310-312, 1995.

[21] L. A. Ferreira and J. L. Santos, "Fiber optic interferometric sensor based on source coherence synthesis by dynamical spectral filtering of a superluminescent source," Optics Communications, vol. 114, no. 5-6, pp. 381-385, 1995.

[22] C. Boulet, M. Hathaway, and D. A. Jackson, "Fiber-optic-based absolute displacement sensors at $1500 \mathrm{~nm}$ by means of a variant of channeled spectrum signal recovery," Optics Letters, vol. 29, no. 14, pp. 1602-1604, 2004.

[23] D. Huang, E. A. Swanson, C. P. Lin, J. S. Schuman, W. G. Stinson, W. Chang, M. R. Hee, T. Flotte, K. Gregory, C. A. Puliafito, and J. G. Fujimoto, "Optical coherence tomography," Science, vol. 254, no. 5035, pp. 1178-1181, 1991.

[24] A. Gh. Podoleanu, G. M. Dobre, and D. A. Jackson, "En-face coherence imaging using galvanometer scanner modulation," Optics Letters, vol. 23, no. 3, pp. 147-149, 1998.

[25] C. C. Rosa, J. Rogers, J. Pedro, R. Rosen, and A. Podoleanu, "Multi-scan time domain OCT for retina imaging," Applied Optics, vol. 46, no. 10, pp. 1795-1808, 2007.

[26] A. Gh. Podoleanu and R. B. Rosen, "Combinations of techniques in imaging the retina with high resolution," Progress in Retinal and Eye Research, vol. 27, no. 4, pp. 464-499, 2008.

[27] A. Gh. Podoleanu and D. A. Jackson, "Combined optical coherence tomograph and scanning laser ophthalmoscope," Electronics Letters, vol. 34, no. 11, pp. 1088-1090, 1998

[28] A. Gh. Podoleanu, G. M. Dobre, R. G. Cucu, R. B. Rosen, P. Garcia, J. Nieto, D. Will, R. Gentile, T. Muldoon, J. Walsh, L. A. Yannuzzi, Y. Fisher, D. Orlock, R. Weitz, J. A. Rogers, S. Dunne, and A. Boxer, "Combined multiplanar optical coherence tomography and confocal scanning ophthalmoscopy," Journal of Biomedical Optics, vol. 9, no. 1, pp. 86-93, 2004.

[29] L. An and R. K. Wang, "Use of a scanner to modulate spatial interferograms for in vivo full-range Fourier-domain optical coherence tomography," Optics Letters, vol. 32, no. 23, pp. 3423-3425, 2007.

[30] A. Gh. Podoleanu and D. A. Jackson, "Noise analysis of a combined optical coherence tomograph and a confocal scanning ophthalmoscope," Applied Optics, vol. 38, no. 10, pp. 2116-2127, 1999.

[31] R. B. Rosen, P. Garcia, A. Gh. Podoleanu, R. G. Cucu, G. Dobre, M. E. J. Van Velthoven, M. D. de Smet, J. A. Rogers, M. Hathaway, J. Pedro, and R. Weitz, "En-face flying spot OCT/ophthalmoscope optical coherence tomography, technology and applications," in series: biological and medical physics, biomedical engineering, Drexler, Wolfgang, Fujimoto, G. James, Eds., 2008, XXVIII, 1357, Berlin Heidelberg: Springer, 2008, pp. 448-474.

[32] A. Gh. Podoleanu, G. M. Dobre, R. Cernat, J. A. Rogers, J. Pedro, R. B. Rosen, and P. Garcia, "Investigations of the eye fundus using a simultaneous optical coherence tomography/indocyanine green fluorescence imaging system," Journal of Biomedical Optics, vol. 12, no. 1, pp. 014019, 2007.

[33] A. Bradu, L. Ma, J. W. Bloor, and A. Podoleanu, "Dual optical coherence tomography/fluorescence microscopy for monitoring of drosophila melanogaster larval hear," J. Biophoton., vol. 2, no. 6-7, pp. 380-388, 2009.

[34] A. Gh. Podoleanu, G. M. Dobre, D. J. Webb, and D. A. Jackson, "Simultaneous en-face imaging of two layers in human retina," Optics Letters, vol. 22, no. 13, pp. 1039-1041, 1997.

[35] A. Gh. Podoleanu, J. A. Rogers, R. C. Cucu, D. A. Jackson, B. Wacogne, H. Porte, and T. Gharbi, "Simultaneous low coherence interferometry imaging at two depths using an integrated optic modulator," Optics Communications, vol. 191, no. 1-2, pp. 21-30, 2001.

[36] L. Plesea and A. Gh. Podoleanu, "Direct corneal elevation measurements using multiple delay en-face OCT," Journal of Biomedical Optics, vol. 13, no. 5, pp. 054054, 2008.

[37] J. Wang, C. Dainty, and A. Podoleanu, "Multiple delay lines full-field optical coherence tomography," Proc. SPIE, vol. 7139, pp. 71390A, 2008. 
[38] A. Gh. Podoleanu, G. M. Dobre, R. G. Cucu, and R. Rosen, "Sequential OCT and confocal imaging," Optics Letters, vol. 29, no. 4, pp. 364-366, 2004.

[39] I. Trifanov, M. Hughes, R. B. Rosen, and A. Gh. Podoleanu, "Quasi-simultaneous OCT/confocal imaging," Journal of Biomedical Optics, vol. 13, no. 4, pp. 044015, 2008.

[40] A. Gh. Podoleanu, J. A. Rogers, and D. A. Jackson, "OCT en-face images from the retina with adjustable depth resolution in real time," IEEE Journal of Selected Topics in Quantum Electronics, vol. 5, no. 4, pp. 1176-1184, 1999.

[41] C. C. Rosa, J. Rogers, and A. Gh. Podoleanu, "Fast scanning transmissive delay line optical coherence tomography," Optics Letters, vol. 30, no. 24, pp. 3263-3265, 2005.

[42] S. R. Taplin, A. Gh. Podoleanu, D. J. Webb, D. A. Jackson, and S. R. Nattrass, Applications of low coherence interferometry to dynamic oil film thickness measurement, Applications of Photon Technology, G. A. Lambropoulos and R. A. Lessard, Eds. New York: Plenum Press, 1997, pp. 863-869.

[43] S. Taplin, A. Gh. Podoleanu, D. J. Webb, and D. A. Jackson, "Displacement sensor using channeled spectrum dispersed on a linear CCD array," Electronics Letters, vol. 29, no. 10, pp. 896-897, 1993.

[44] A. Gh. Podoleanu, S. Taplin, D. J. Webb, and D. A. Jackson, "Channelled spectrum liquid refractometer," Rev. Sci. Instr., vol. 64, no. 10, pp. 3028-3029, 1993.

[45] A. Gh. Podoleanu, S. Taplin, D. J. Webb, and D. A. Jackson, "Channeled spectrum display using a CCD array for student laboratory demonstrations," European J. Phys., vol. 15, no. 5, pp. 266-271, 1994.

[46] A. Gh. Podoleanu, S. Taplin, D. J. Webb, and D. A. Jackson, "Theoretical study of Talbot-like bands observed using a laser diode below threshold," Pure and Applied Optics: Journal of the European Optical Society Part A, vol. 7, no. 3, pp. 517-536, 1998.

[47] A. Gh. Podoleanu, S. Taplin, D. J. Webb, and D. A. Jackson, "Talbot-like bands for laser diode below threshold," Pure and Applied Optics: Journal of the European Optical Society Part A, vol. 6, no. 3, pp. 413-424, 1997.

[48] A. Gh. Podoleanu, "Unique interpretation of Talbot bands and Fourier domain white light interferometry," Optics Express, vol. 15, no. 15, pp. 9867-9876, 2007.

[49] D. Woods and A. Gh. Podoleanu, "Controlling the shape of Talbot bands' visibility," Optics Express, vol. 16, no. 13, pp. 9654-9670, 2008.

[50] A. Podoleanu and D. Woods, "Power-efficient Fourier domain optical coherence tomography setup for selection in the optical path difference sign using
Talbot bands," Optics Letters, vol. 32, no. 16, pp. 2300-2302, 2007.

[51] M. Hughes, D. Woods, and A. Gh. Podoleanu, "Control of visibility profile in spectral low-coherence interferometry," Electronics Letters, vol. 45 no. 3, pp. 182-183, 2009.

[52] R. G. Cucu, A. Gh. Podoleanu. A. Rogers, J. Pedro, and R. B. Rosen, "Combined confocal scanning ophthalmoscopy/en face T-scan based ultrahigh resolution OCT of the human retina in vivo," Optics Letters, vol. 31, no. 11, pp. 1684-1687, 2006.

[53] R. G. Cucu, J. A. Rogers, M. W. Hathaway J. Pedro, A. Gh. Podoleanu, and R. B. Rosen, "Combined confocal/en face optical coherence tomography imaging of the human eye fundus in vivo in the 1050 nm spectral region," Proc. SPIE, vol. 6429, pp. 642903-01-642903-05, 2007.

[54] A. Gh. Podoleanu, J. A. Rogers, D. A. Jackson, and S. Dunne "Three dimensional OCT images from retina and skin," Optics Express, vol. 7, no. 9, pp. 292-298, 2000.

[55] M. Khandwala, B. R. Penmetsa, S. Dey, J. B. Schofield, C. A. Jones, and A. Podoleanu, "Imaging of periocular basal cell carcinoma using en face optical coherence tomography: a pilot study," Clinical science: Br J Ophthalmol, vol. 94, no. 10, pp. 1332-1336, 2010.

[56] A. G. Bibas, A. Gh. Podoleanu, R. G. Cucu, M. Bonmarin, G. M. Dobre, V. M. M Ward, E. Odell, A. Boxer, M. L Harries, and M. J. Gleeson, “3-D optical coherence tomography of the laryngeal mucosa," Clinical Otolaryngology, vol. 29, no. 6, pp. 713-720, 2004.

[57] B. T. Amaechi, A. Gh. Podoleanu, S. M. Higham, and D. A. Jackson, "Correlation of quantitative light induced fluorescence and optical coherence tomography applied for detection and quantification of early dental caries," Journal Biomedical Optics, vol. 8, no. 4, pp. 642-647, 2003.

[58] C. D. Russell, K. Kosmidis, R. A. Black George Dobre, and A. Gh. Podoleanu, "Flying spot en-face OCT for monitoring cell distribution in collagen-based constructs," Proc. SPIE, vol. 6079, pp. 60790Q-1-60790Q-5, 2006.

[59]H. Liang, M. G. Cid, R. Cucu, G. M. Dobre, J. Pedro, D. Saunders, and A. Gh. Podoleanu, "Application of optical coherence tomography to examination of easel paintings," Optics Express, vol. 13, no. 16, pp. 6133-6144, 2005.

[60] M. Hughes, M. Spring, and A. Podoleanu, "Speckle noise reduction in optical coherence tomography of paint layers," Applied Optics, vol. 49, no. 1, pp. 99-107, 2010.

[61] C. C. Rosa, J. Rogers, and A. Gh. Podoleanu, "Fast scanning transmissive delay line optical coherence tomography," Optics Letters, vol. 30, no. 24, pp. 3263-3265, 2005. 
[62] M. Hughes and A. Gh. Podoleanu, "Simplified dynamic focus method for time domain OCT," Electronics Letters, vol. 45, no. 12, pp. 623-624, 2009.

[63] R. G. Cucu, M. W. Hathaway, A. Gh. Podoleanu, and R. B. Rosen, "Variable lateral size imaging of the human retina in vivo by combined confocal/ en face optical coherence tomography with closed loop OPD-locked low coherence interferometry based active axial eye motion tracking," Proc. SPIE, vol. 7554, pp. 75540J, 2010.

[64] A. Gh. Podoleanu, "Unbalanced versus balanced operation in an OCT system," Applied Optics, vol. 39, no. 1, pp. 173-182, 2000.

[65] A. Gh. Podoleanu and D. A. Jackson, "Noise analysis of a combined optical coherence tomograph and a confocal scanning ophthalmoscope," Applied Optics, vol. 38, no. 10, pp. 2116-2127, 1999.

[66] C. C. Rosa and A. Podoleanu, "Limitation of the achievable signal to noise ratio in OCT due to mismatch of the balanced receiver," Applied Optics, vol. 43, no. 25, pp. 4802-4815, 2004.

[67] S. Sherif, C. Rosa, C.Flueraru, S. Chang, Y. Mao, and A. Gh. Podoleanu, "Statistics of the depth-scan photocurrent in time-domain optical coherence tomography," J. Opt. Soc. Am. A, vol. 25, no.1, pp.16-20, 2008.

[68] A. Gh. Podoleanu, R. G. Cucu, and D. A. Jackson, "Signal to noise ratio in an OCT/confocal system and penetration depth in OCT," Proc. SPIE, vol. 4251, pp. 11-20, 2001.

[69] D. Merino, C. Dainty, A. Bradu, and A. Gh. Podoleanu, "Adaptive optics enhanced simultaneous en-face optical coherence tomography and scanning laser ophthalmoscopy," Optics Express, vol. 14, no. 8, pp. 3345-3353. 2006.

[70] S. Tuohy and A. Gh. Podolean, "Depth-resolved wavefront aberrations using a coherence-gated Shack-Hartmann wavefront sensor," Optics Express, vol. 18, no. 4, pp. 3458-3476, 2010. 